International Journal of Pure and Applied Mathematics

Volume 103 No. 1 2015, 81-97

ISSN: 1311-8080 (printed version); ISSN: 1314-3395 (on-line version)

url: http://www.ijpam.eu

doi: http://dx.doi.org/10.12732/ijpam.v103i1.7

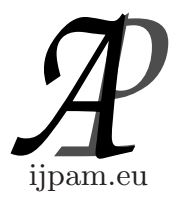

\title{
FLOW REVERSAL OF FULLY DEVELOPED MIXED CONVECTION OF NANOFLUIDS IN A VERTICAL CHANNEL FILLED WITH POROUS MEDIUM
}

\author{
Nur Asiah Mohd Makhatar ${ }^{1}$, Habibis Saleh ${ }^{1}$, Ishak Hashim ${ }^{1,2}$ \\ ${ }^{1}$ School of Mathematical Sciences \\ Universiti Kebangsaan Malaysia \\ 43600, UKM Bangi Selangor, MALAYSIA \\ ${ }^{2}$ Solar Energy Research Institute \\ Universiti Kebangsaan Malaysia \\ 43600, UKM Bangi Selangor, MALAYSIA
}

\begin{abstract}
The present analysis is concerning the criteria for the onset of flow reversal of the fully developed flow mixed convection of nanofluids in a vertical channel filled with porous medium. The governing equations and the critical values of the buoyancy force are solved and calculated numerically via dsolve package in MAPLE. It was found that the critical values of mixed convection parameter for the occurrence of reversed flow decreases with increasing temperature difference ratio and increases with increasing nanoparticles mass flux.
\end{abstract}

AMS Subject Classification: 76S99, 12D05

Key Words: mixed convection, vertical channel, flow reversal, nanofluids

\section{Introduction}

Mixed convection are coexistence of forced and natural convection. The study

Received: March 18, 2015

(C) 2015 Academic Publications, Ltd.

${ }^{\S}$ Correspondence author url: www.acadpubl.eu 
of mixed convection flow in a heated vertical parallel-plate channel filled with porous medium has received considerable attention because of its wide range of applications such as geothermal system, cooling of nuclear reactors, thermal insulation, energy storage and conservation, chemical, food and metallurgical industries petroleum reservoirs and operations of electronic devices. One of the earliest studies on mixed convection in a vertical channel with asymmetric wall heating had been studied by [9]. They have modeled the porous layer using non-Darcy. [3] included the heat generation, absorption and hydromagnetics effects. [14] studied the effect of inertial forces by taking into account the effect of viscous and Darcy dissipations. The flow is modeled using the BrinkmanForchheimer-extended Darcy equations. Later [13] studied the problem with boundary conditions of third kind.

The volcanic eruption, material fabrication, cooking and transportation utilizing internal combustion and jet engines, unintentionally release nanoparticles into the atmosphere. These nanoparticles dispersed in the pure fluid such as water known as nanofluids. In recent years a new type of nanoparticles source coming form nanotechnology has been introduced, and followed by an engineered nanofluids, product of [6]. Nanofluids in vertical porous media with viscous heating had been studied by [12]. [10] and [11] studied nanofluids in a vertical channel filled with highly porous medium. They found that flow reversal at the cold wall of the channel becomes smaller for nanofluids compared to regular fluids and the flow reversal extents by mixed convection parameter. However, they overlooked the effects of nanofluids combined with the porous medium properties in determining the criteria for the onset of flow reversal. Therefore, in this article, we present the conditions for the onset of flow reversal.

The high buoyancy force due to a differentially heated wall combined with an upward flow leads to a high fluid flow adjacent to the walls that can precipitate a downward flow (i.e. reversal flow) emanating from the open top of the channel in order to augment the increased upward flow. Theory on the appearance of flow reversal was conducted by [1] and [5] for mixed convection in vertical channel filled with regular fluids. Criteria for the occurrence of reversal flow, adjacent to the colder wall under effects of concentration, magnetic field including internal heating, micropolar as well as thermophoretic were presented by [7], [2], [4] and [8], respectively. 


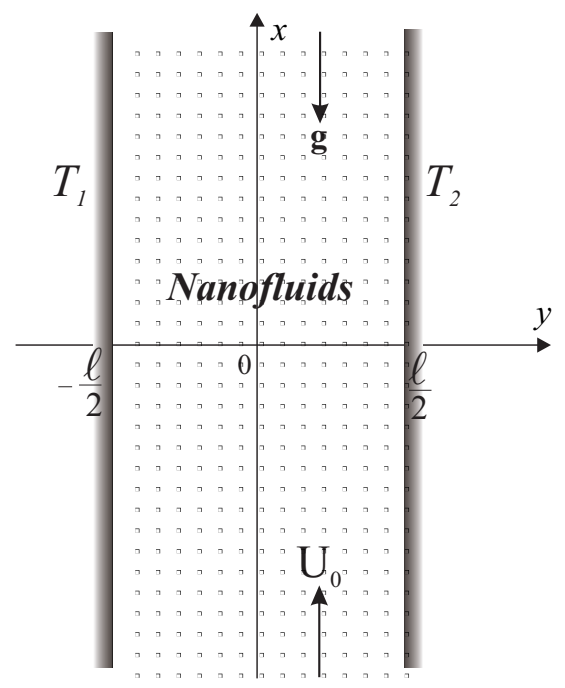

Figure 1: Schematic representation of the model.

\section{Mathematical Formulation}

Consider the steady flow of a viscous and incompressible nanofluids between two vertical and parallel plane walls. The distance between the walls, i.e., the channel width, is $\ell$. A coordinate system is chosen such that the $x$-axis is parallel to the gravitational acceleration vector $\mathbf{g}$, but with the opposite direction. The $y$-axis is orthogonal to the channel walls, and the origin of the axes is such that the positions of the channel walls are $y=-\ell / 2$ and $y=\ell / 2$, respectively. A sketch of the system and of the coordinate axes is reported in Fig. 1. The walls at $y=-\ell / 2$ and $y=\ell / 2$ are isothermal at given temperatures $T_{1}$ and $T_{2}$, where we assume that $T_{1} \geq T_{2}$. The nanofluids has a uniform vertical upward stream wise velocity distribution $U_{0}$ at the channel entrance. The Boussinesq approximation is employed and homogeneity and local thermal equilibrium in the porous medium are considered. The nanofluids is assumed to saturate the solid matrix and both are in thermodynamic equilibrium. The Brinkman-Forchheimer extended Darcy equation was adopted for the porous layer. Based on these assumptions and taking into account the effect of viscous dissipation,the following relations apply:

$$
v=0, \quad \frac{\partial u}{\partial x}=0, \quad \frac{\partial T}{\partial x}=0
$$




$$
\frac{\partial P}{\partial x}=\text { const. }=A, \quad \frac{\partial P}{\partial y}=0
$$

in which $v$ is the velocity component in the $y$-direction and $P$ is the pressure. This will lead us to the governing equations for fully developed flow:

$$
\begin{aligned}
& g \beta\left(T-T_{0}\right)-\frac{1}{\rho} \frac{\mathrm{d} p}{\mathrm{~d} x}+\nu_{\text {eff }} \frac{\mathrm{d}^{2} u}{\mathrm{~d} y^{2}}-\frac{\nu}{K} u-\frac{C_{F}}{\sqrt{K}} u^{2}=0 \\
& k \frac{\mathrm{d}^{2} T}{\mathrm{~d} y^{2}}+\epsilon \zeta\left(\rho C_{p}\right)_{s p} \frac{\mathrm{d} T}{\mathrm{~d} y}+\Phi=0
\end{aligned}
$$

where $T_{0}$ is the reference temperature and the coefficient $v_{\text {eff }}=v_{1} /$ isthe effective viscosity of fluid in the porous region. Meanwhile, $\zeta$ and $\Phi$ are respectively a constant and the viscous heating due to viscous dissipation [10]. They are defined as:

$$
\begin{aligned}
& \zeta=D_{B} \frac{\mathrm{d} \phi}{\mathrm{d} y}+\frac{D_{T}}{T} \frac{\mathrm{d} T}{\mathrm{~d} y} \\
& \Phi=\frac{\mu}{K} u^{2}-\mu\left(\frac{\mathrm{d} u}{\mathrm{~d} y}\right)^{2}+\frac{\rho C_{F}}{\sqrt{K}} u^{3}
\end{aligned}
$$

The walls of the channel are assumed isothermal with no-slip conditions:

$$
\begin{aligned}
& u\left(-\frac{\ell}{2}\right)=u\left(\frac{\ell}{2}\right)=0 \\
& \left.\frac{\mathrm{d}^{2} u}{\mathrm{~d} y^{2}}\right|_{y=-\frac{\ell}{2}}=-\frac{A}{\mu_{\mathrm{eff}}}+\frac{g \beta \rho\left(T_{1}-T_{0}\right)}{\mu_{\mathrm{eff}}}, \\
& \left.\frac{\mathrm{d}^{2} u}{\mathrm{~d} y^{2}}\right|_{Y=\frac{\ell}{2}}=-\frac{A}{\mu_{\mathrm{eff}}}-\frac{g \beta \rho\left(T_{2}-T_{0}\right)}{\mu_{\mathrm{eff}}} \\
& T\left(-\frac{\ell}{2}\right)=T_{1}, \quad T\left(\frac{\ell}{2}\right)=T_{2}, \quad\left(T_{1} \geq T_{2}\right)
\end{aligned}
$$

With reference to equation (2), the first and second derivatives of $T$ with respect to $y$ is substituted into the thermal energy equation, i.e. equation (3).Hence, we obtain:

$$
\begin{aligned}
& \frac{\mathrm{d}^{4} u}{\mathrm{~d} y^{4}}=-\frac{\varepsilon\left(\rho C_{p}\right)_{s p} \zeta}{k} \frac{\mathrm{d}^{3} u}{\mathrm{~d} y^{3}}+\frac{v}{v_{\text {eff }} K} \frac{\mathrm{d}^{2} u}{\mathrm{~d} y^{2}}+\frac{\varepsilon\left(\rho C_{p}\right)_{s p} \zeta v}{K k v_{\text {eff }}} \frac{\mathrm{d} u}{\mathrm{~d} y}+\frac{\mathbf{g} \beta \mu}{K k v_{\text {eff }}} u^{2} \\
& +\left(\frac{2 C_{F} \varepsilon\left(\rho C_{p}\right)_{s p} \zeta}{\sqrt{K} k v_{\text {eff }}}\right) u \frac{\mathrm{d} u}{\mathrm{~d} y}-\left(\frac{2 C_{F}}{v_{\text {eff }} \sqrt{K}}-\frac{\mathbf{g} \beta \mu}{k v_{\text {eff }}}\right)\left(\frac{\mathrm{d} u}{\mathrm{~d} y}\right)^{2}
\end{aligned}
$$




$$
+\frac{2 C_{F}}{v_{\text {eff }} \sqrt{K}} u \frac{\mathrm{d}^{2} u}{\mathrm{~d} y^{2}}+\frac{\mathbf{g} \rho C_{F} \rho}{k v_{\text {eff }} \sqrt{K}}=0
$$

The following dimensionless parameters were applied to transform these equations into dimensionless forms:

$$
\begin{aligned}
& \Theta=\frac{T-T_{0}}{\Delta T}, \quad Y=\frac{y}{L}, \quad U=\frac{u \mu}{A h^{2}}, \quad G r=\frac{\mathbf{g} \beta \Delta T L^{3}}{v^{2}}, \quad G_{R}=\frac{G r}{R e} \\
& R e=\frac{A L^{3} \rho}{\mu^{2}}, \quad B r=\frac{A^{2} L^{4}}{\mu k \Delta T}, \quad D a=\frac{K}{L^{2}}, \quad M=\frac{\mu_{e f f}}{\mu}, \quad \sigma=\frac{1}{\sqrt{D a M}} \\
& N=\frac{\varepsilon\left(\rho C_{p}\right)_{s p} \zeta L}{k}, \quad F=\frac{C_{F} \rho L^{4} A}{\mu^{2} \sqrt{K}}, \quad \Delta T=T_{2}-T_{1}, \quad T_{0}=\frac{T_{1}+T_{2}}{2}
\end{aligned}
$$

Using these dimensionless parameters, the dimensionless governing equations for the problem are:

$$
\begin{aligned}
& \frac{\mathrm{d}^{4} U}{\mathrm{~d} Y^{4}}+N \frac{\mathrm{d}^{3} U}{\mathrm{~d} Y^{3}}-\frac{1}{D a M} \frac{\mathrm{d}^{2} U}{\mathrm{~d} Y^{2}} \\
& -N \frac{1}{D a M} \frac{\mathrm{d} U}{\mathrm{~d} Y}-\frac{2 F}{M}\left[\left(\frac{\mathrm{d} U}{\mathrm{~d} Y}\right)^{2}+U \frac{\mathrm{d}^{2} U}{\mathrm{~d} Y^{2}}+N U \frac{\mathrm{d} U}{\mathrm{~d} Y}\right] \\
& -G_{R} B r\left[\frac{1}{D a M} U^{2}+\frac{1}{M}\left(\frac{\mathrm{d} U}{\mathrm{~d} Y}\right)^{2}+\frac{F}{M} U^{3}\right]=0 \\
& \frac{\mathrm{d}^{2} \Theta}{\mathrm{d} Y^{2}}+N \frac{\mathrm{d} \Theta}{\mathrm{d} Y}+\frac{B r}{M}\left[\frac{1}{D a M} U^{2}+\left(\frac{\mathrm{d} U}{\mathrm{~d} Y}\right)^{2}+F U^{3}\right]=0
\end{aligned}
$$

subject to the boundary conditions:

$$
\begin{aligned}
& U\left(-\frac{1}{4}\right)=U\left(\frac{1}{4}\right)=0 \\
& \left.\frac{\mathrm{d}^{2} U}{\mathrm{~d} Y^{2}}\right|_{Y=-\frac{1}{4}}=-\frac{48}{M}+\frac{R_{T} G_{R}}{2 M},\left.\quad \frac{\mathrm{d}^{2} U}{\mathrm{~d} Y^{2}}\right|_{Y=\frac{1}{4}}=-\frac{48}{M}-\frac{R_{T} G_{R}}{2 M} \\
& \Theta\left(-\frac{1}{4}\right)=-\frac{R_{T}}{2}, \quad \Theta\left(\frac{1}{4}\right)=\frac{R_{T}}{2}
\end{aligned}
$$

Here, $B r$ is the Brinkman number, $R_{T}$ is the temperature difference ratio, $G_{R}=G r / R e$, where $G r$ is the Grashof number and Re is the Reynolds number. 


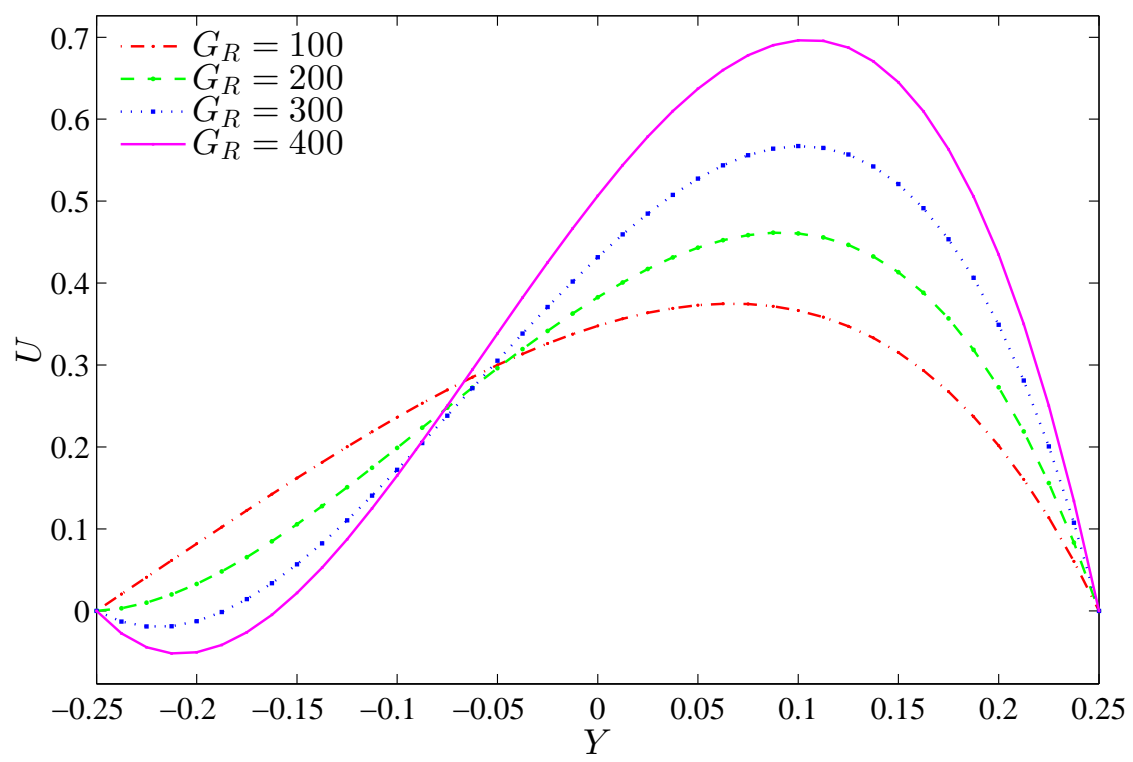

Figure 2: Plots $U$ versus $Y$ for different values of $G_{R}$ at $R_{T}=1$, $B r=0.05, D a=0.01, F=1$ and $N=0.5$.

\section{Solutions Method}

System (11) and (12) subject to the boundary conditions (14)-(16) is a kind of nonlinear boundary value problem (BVP) for which no available exact solutions have been reported. Therefore, the BVP will be solved numerically by dsolve from Maple. The nonlinearities was handled by applying continuation, optional in dsolve. The continuation is utilized to maintain Newton method (inside package dsolve) to reach the convergence. Custom user interfaces in Maple could be created. Maple is a commercial computer algebra system developed and sold commercially by Maplesoft, a software company also based in Waterloo, Ontario, Canada. We use Maple version 16, which is an improvement from its predecessor Maple 15.

\section{Results and Discussion}

Figure 2 shows the dimensionless velocity for different values of $G_{R}$. The flow velocity increases in the right portion and decreases in the left portion of channel 


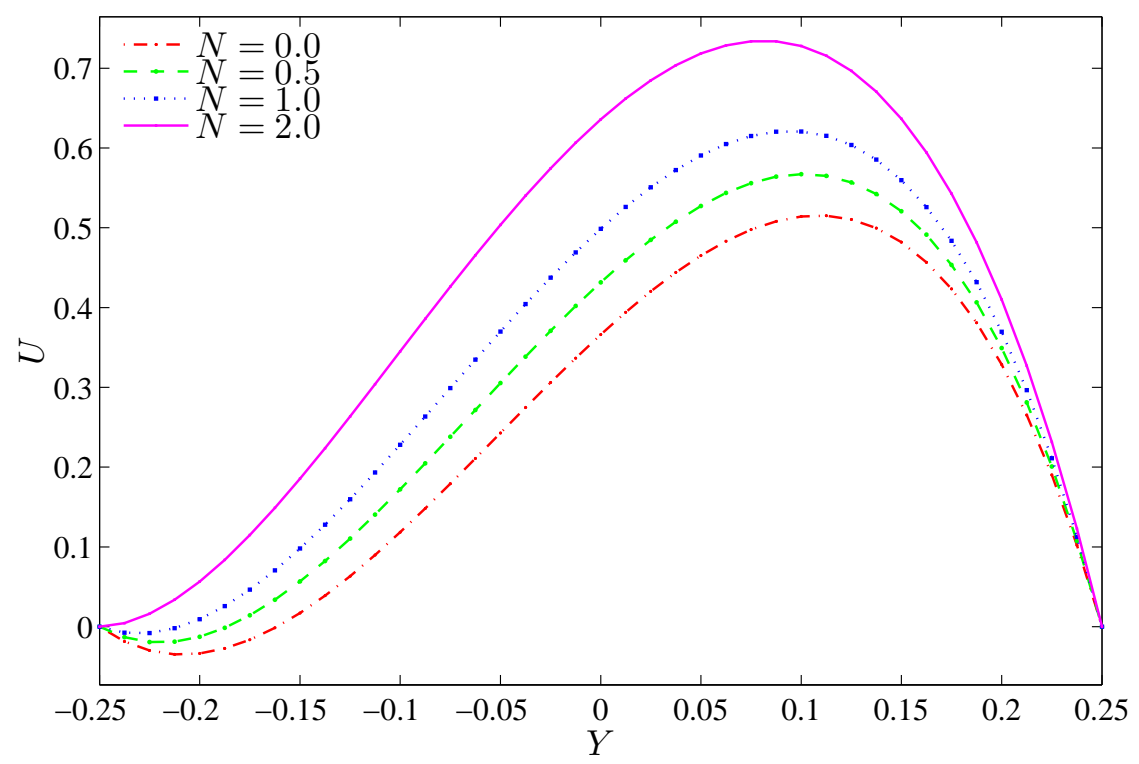

Figure 3: Plots of $U$ versus $Y$ for different values of $N$ at $G_{R}=300$, $R_{T}=1, D a=0.01, F=1$ and $B r=0.05$.

as mixed convection parameter (i.e., $G_{R}$ ) increase. Mixed convection parameter is also a representation of the ratio of natural convection to forced convection. For small values of mixed convection parameter, the forced convection flow is dominant and for very large values of $G_{R}$ the natural convection flow is dominant in the channel. As the parameter $G_{R}$ exceeds 200, the velocity in the left portion become negative so that flow reversal appears along the cold wall and fluid moves downward in the region close to the cold wall. The variation of other parameters at $G_{R}=300$ to velocity especially for the occurrence of reversed flow would be investigated next.

The effects of various levels $N$ on dimensionless velocity is presented in Figure 3. Increasing dimensionless parameter for nanoparticles mass flux increases the velocity in the whole channel. The maximum velocity location moves to the middle channel as the nanoparticles mass flux get stronger. Increasing the nanoparticles mass flux parameter shrinks the flow reversal near the colder wall. Furthermore, flow reversal vanishes as nanoparticles mass flux parameter reaches 2.0. This behavior can be explained by greater heat transmission due to the nanoparticles mass flux, which increases the fluid temperature in the channel, with the consequence of a greater buoyancy force at any loca- 


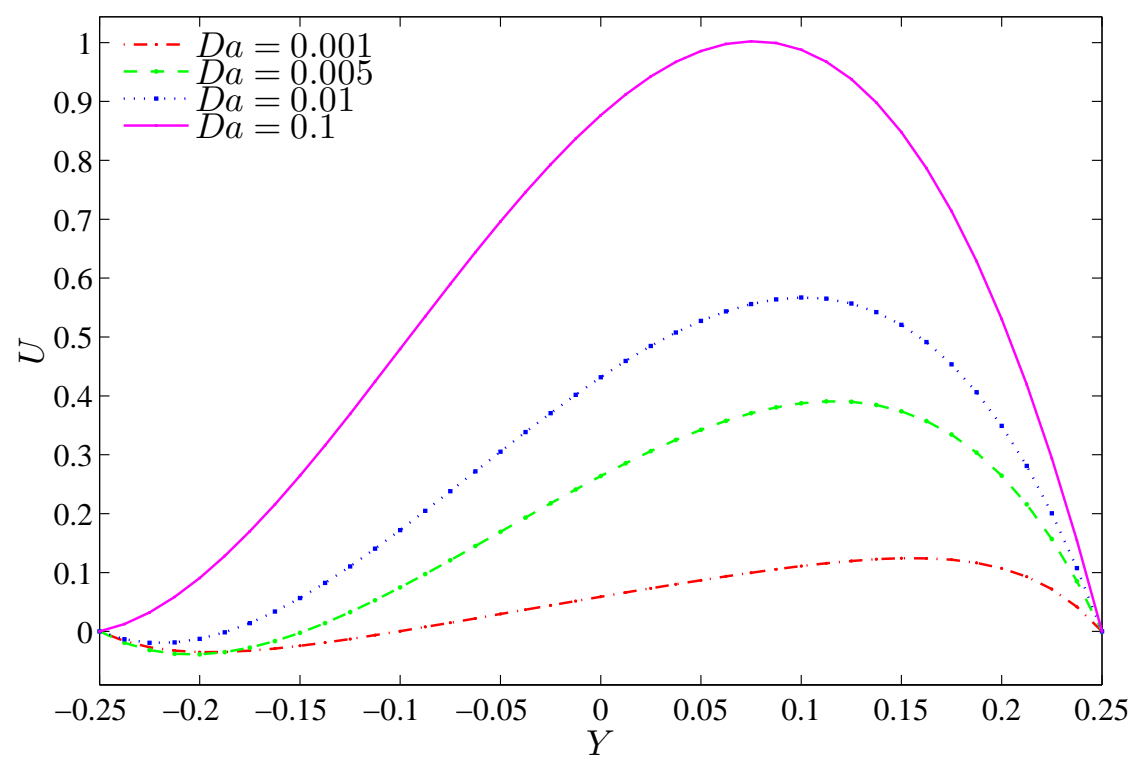

Figure 4: Plots of $U$ versus $Y$ for different values of $D a$ at $G R=300$, $R_{T}=1, B r=0.05, F=1$ and $N=0.5$.

tion. Therefore, an increase in the buoyancy force increases the velocity in the upward direction.

The effects of various levels of Darcy number on dimensionless velocity is presented in Figure 4. Obviously, increasing $D a$ increases the velocity. The maximum velocity location moves to the middle channel as the Darcy number get higher. Flow reversal is found near the colder wall for $D a<0.1$. Generally, decreasing the Darcy number enhances the flow reversal and suppress the maximum velocity near the hotter wall. It is observed at $D a=0.001$ that the flow reversal is slightly smaller compared to the flow reversal at $D a=0.005$. This means that a sufficiently low Darcy number weakens the velocity at each position including flow reversal near the colder wall.

Figure 5 presents effects of various levels of Brinkman number on dimensionless velocity. Obviously, increasing viscous dissipation increases the velocity and decrease the flow reversal. This fact is because of a greater energy generated by viscous dissipation yields a greater fluid temperature that leads to increasing the buoyancy force at each position and as a consequence it tends to contrast the flow reversal. Flow reversal occurs only for a large mixed convection parameter combined with low nanoparticles mass flux parameter, low Darcy number 


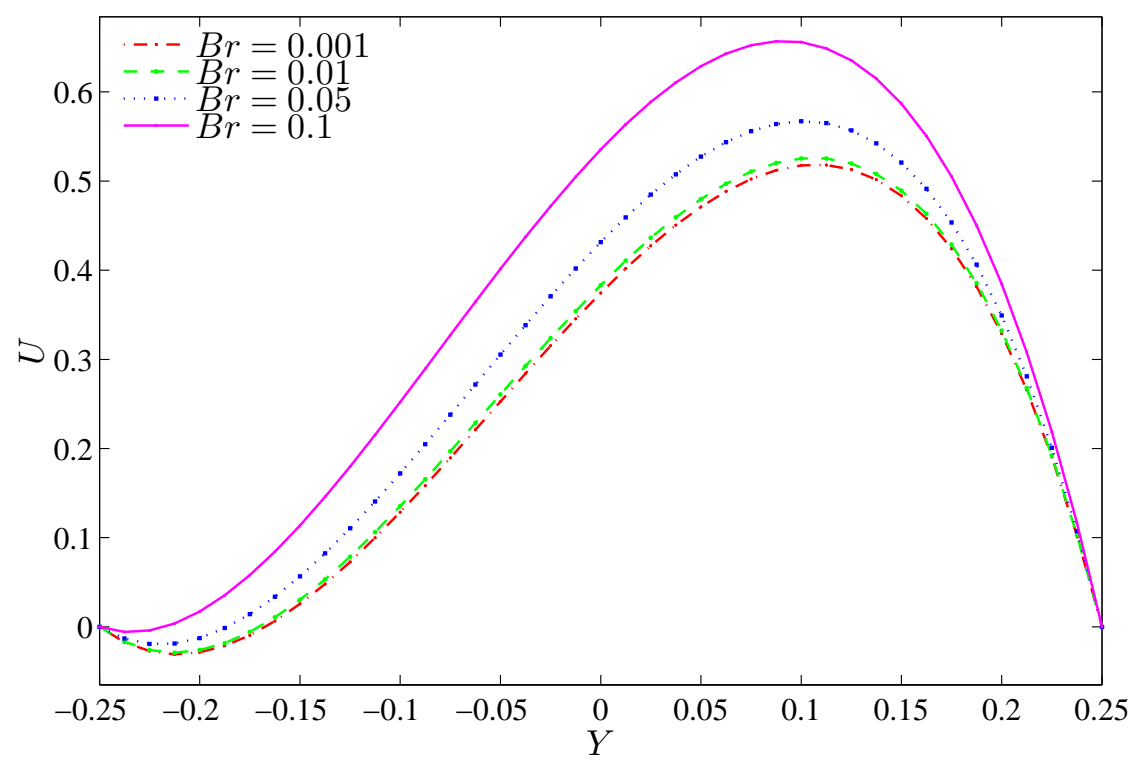

Figure 5: Plots of $U$ versus $Y$ for different values of $B r$ at $G R=300$, $R_{T}=1, D a=0.01, F=1$ and $N=0.5$.

and small Brinkman number. Parameter zones for the occurrence of reversed flow would be presented next.

Figure 6 shows the critical value $G_{R c}$ for the onset of a flow reversal with $N$ for different $B r$ at $R_{T}=1, D a=0.01$ and $F=1$. It was observed that increasing the nanoparticles mass flux increases $G_{R c}$. It notes that $N=0$ refers to regular fluids. The effect of variation $B r$ on $G_{R c}$ nanofluids $(N=2.0)$ is more profound than $G_{R c}$ regular fluid. Brinkman number $(B r)$ represents the effect of heat dissipation in the medium such that large values of $\mathrm{Br}$ show that more heat dissipates in the medium. Heat dissipation can act as a heat source in the medium and raises the nanofluids temperature.

Figure 7 shows the critical value $G_{R c}$ for the onset of a flow reversal with $N$ for different $D a$ at $R_{T}=1, B r=0.05$ and $F=1$. Increasing the nanoparticles mass flux increases $G_{R c}$ for the considered $D a$. The $G_{R c}$ increases by increasing $D a$ at fixed nanoparticles mass flux parameter. This is due to the fluid velocity increases with an increase in the porous medium permeability (i.e., $D a$ increases). In fact, with an increase in the permeability of the porous medium at a fixed pressure gradient, more fluid can pass through the porous medium, thus, the velocity of the nanofluids increases. 


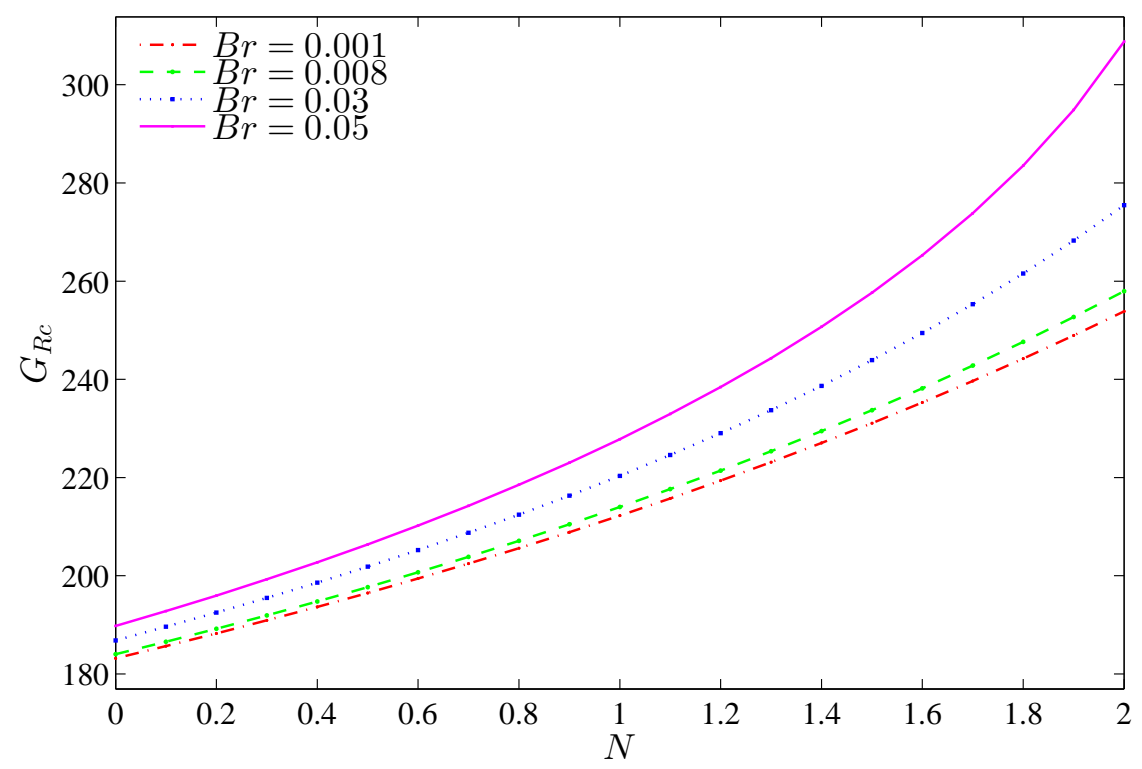

Figure 6: The critical value $G_{R c}$ for the onset of a flow reversal with $N$ for different $B r$ at $R_{T}=1, D a=0.01$ and $F=1$.

Figure 8 shows the critical value $G_{R c}$ for the onset of a flow reversal with $N$ for different $F$ at $R_{T}=1, B r=0.05$ and $D a=0.01$. Increasing the nanoparticles mass flux increases $G_{R c}$ significantly for the considered Forchheimer number. The $G_{R c}$ decreases as Forchheimer number increases at fixed $N$. The Forchheimer number represents the inertial effects in the porous media. The presence of porous medium in the channel increases the flow resistance. In addition, the inertial effect by increasing Forchheimer number assist this resistance, which further decreases the buoyancy force.

Figure 9 shows the critical value $G R_{c}$ for the onset of a flow reversal with $R_{T}$ for different $N$ at $F=1, B r=0.05$ and $D a=0.01$. We limited the $R_{T}$ range from 0.6 to 1 because of $G_{R c}$ exists only in this range for $N=1$. It notes that symmetrical wall temperatures situation when $R_{T}=0$ and at this condition, the flow reversal is impossible to occur in the channel. We observed from Fig. 9 that increasing the temperature difference ratio decreases $G_{R c}$ for all $N$. In particular, the $G_{R c}$ dives in $0.6<R_{T}<0.625$. This fact due to lower degree of the walls temperature variation leading to prevent the occurrence of down flow. 


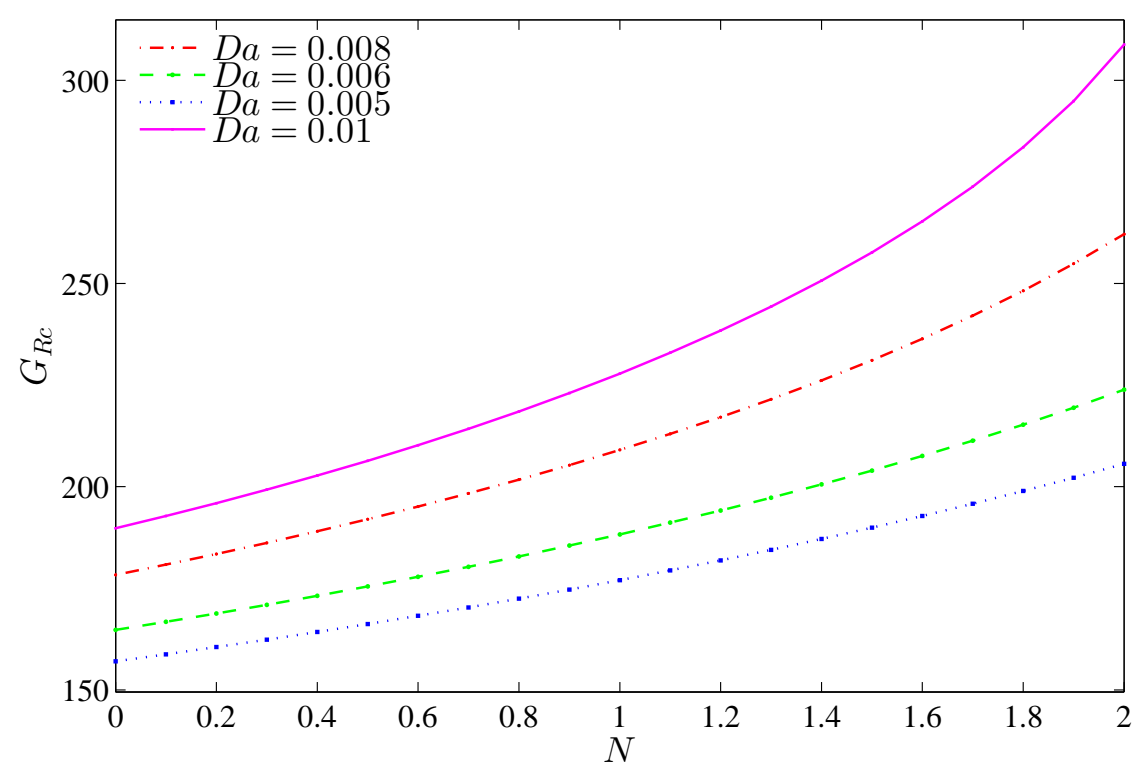

Figure 7: The critical value $G R_{c}$ for the onset of a flow reversal with $N$ for different $D a$ at $R_{T}=1, B r=0.05$ and $F=1$.

Figure 10 shows the critical value $G R_{c}$ for the onset of a flow reversal with $R_{T}$ for different $F$ at $N=1, B r=0.05$ and $D a=0.01$. Increasing the $R_{T}$ decrease $G_{R c}$ for all $F$. $G_{R c}$ decreases significantly in $0.6<R_{T}<0.625$ for $F=1$ while $G_{R c}$ decreases significantly in $0.625<R_{T}<0.65$ for $F=$ $4,8,16$. Variation $G_{R c}$ by adjusting the Forchheimer number is insignificant for $R_{T} \geq 0.6$. Therefore, neglecting the inertial effect (Forchheimer number) in high temperature difference ratio could not cause significant errors.

\section{Conclusions}

The present numerical simulation study the effects of nanofluids combined with the porous medium properties in determining the criteria for the onset of flow reversal. The dimensionless forms of the governing equations are solved using MAPLE. The main conclusions of the present analysis are as follows:

1. Flow reversal occurs only for a sufficiently large mixed convection parameter combined with low nanoparticles mass flux parameter, low Darcy 


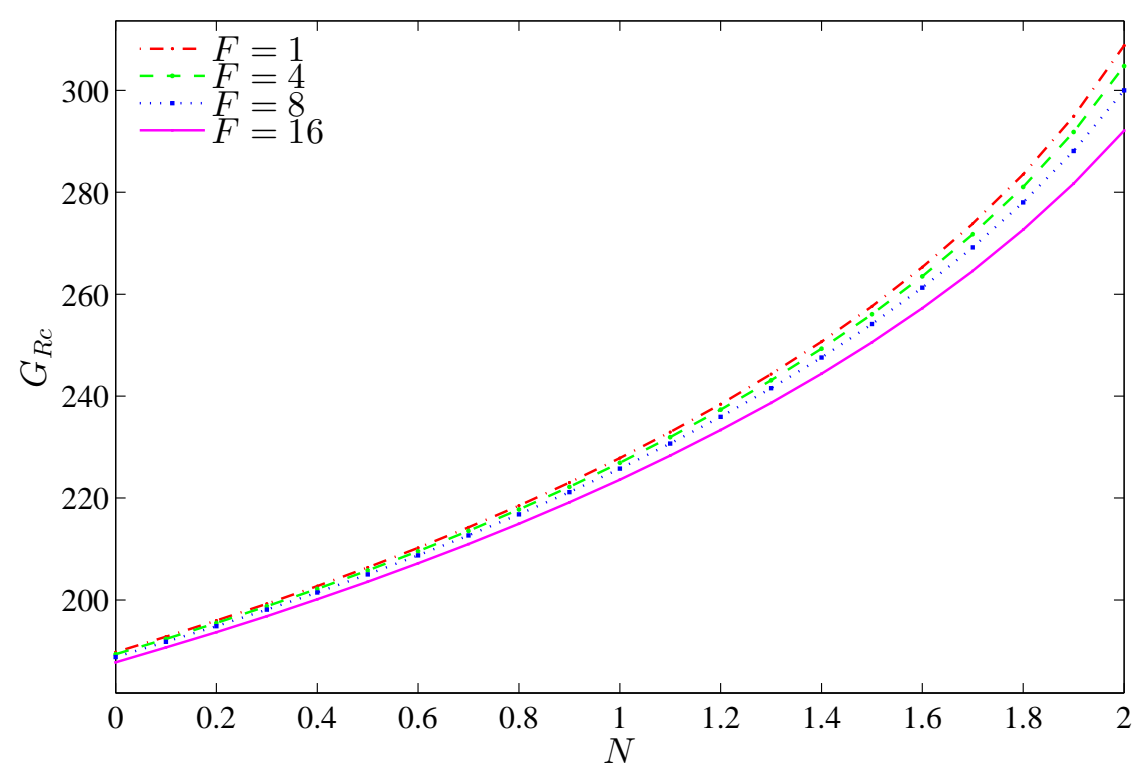

Figure 8: The critical value $G R_{c}$ for the onset of a flow reversal with $N$ for different $F$ at $R_{T}=1, B r=0.05$ and $D a=0.01$.

number and small Brinkman number.

2. The critical values of mixed convection parameter for the occurrence of reversed flow decreases with increasing temperature difference ratio and increases with increasing nanoparticles mass flux.

3. A sufficiently low Darcy number weakens the velocity at each position including flow reversal. Significant effect of Forchheimer number to the onset of a flow reversal was obtained only at high nanoparticles mass flux and low temperature difference ratio. 


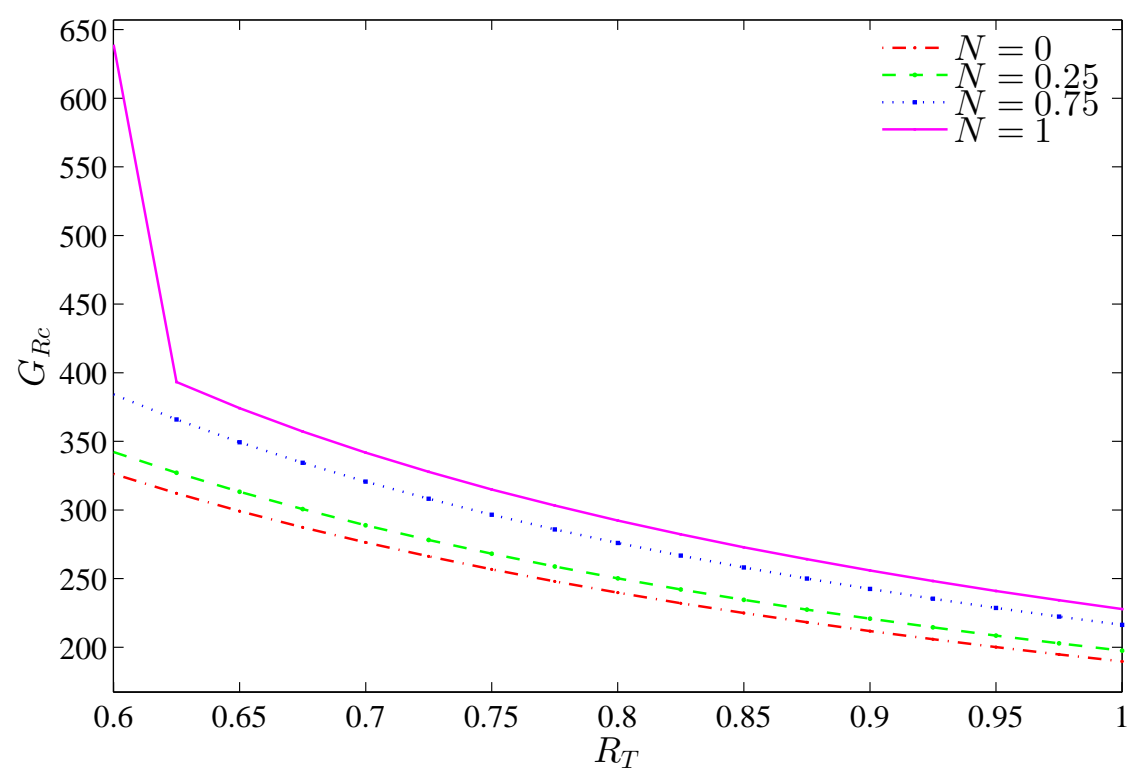

Figure 9: The critical value $G R_{c}$ for the onset of a flow reversal with $R_{T}$ for different $N$ at $F=1, B r=0.05$ and $D a=0.01$. 


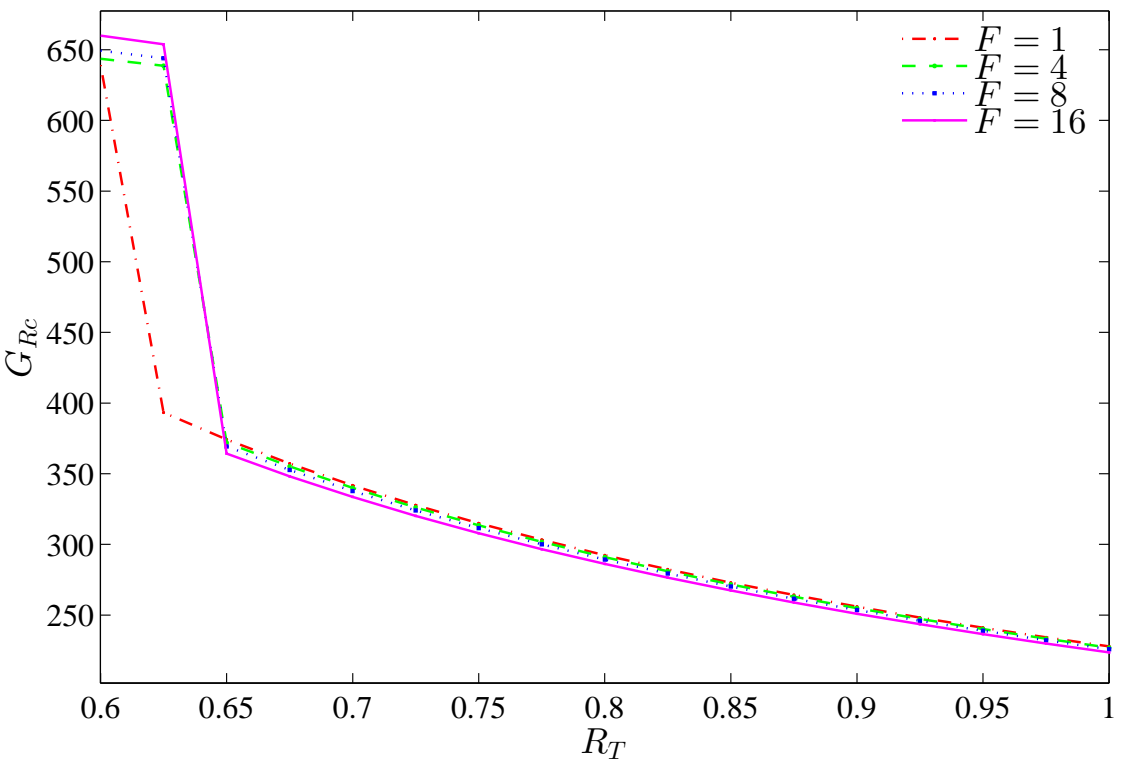

Figure 10: The critical value $G R_{c}$ for the onset of a flow reversal with $R_{T}$ for different $F$ at $N=1, B r=0.05$ and $D a=0.01$. 
Nomenclature

A axial pressure gradient, $\mathrm{d} p / \mathrm{d} x$

$\mathrm{Br} \quad$ Brinkman number

$C_{F} \quad$ inertial coefficient

$C p \quad$ specific heat capacity

Da Darcy number

$F \quad$ Forchheimer number

Gr Grashof number

$G_{R} \quad$ dimensionless parameter, $G r / R e$

$g \quad$ gravitational acceleration

$k \quad$ thermal conductivity

$K \quad$ permeability of porous medium

$N$ dimensionless parameter for nanoparticles mass flux

Re Reynolds number

$R_{T} \quad$ temperature difference ratio

$T$ temperature

$U$ dimensionless velocity components in the $x$-direction

$X, Y$ dimensionless space coordinates

Greek symbols

$\alpha \quad$ thermal diffusivity

$\beta \quad$ thermal expansion coefficient

$\rho$ density

$\Theta \quad$ dimensionless temperature

$\mu \quad$ dynamic viscosity

$\nu \quad$ kinematic viscosity

\section{Subscript}

0 reference value

1 left wall

2 right wall

c critical

bf base fluid

$n f \quad$ nanofluid

$s p \quad$ solid nanoparticles 


\section{Acknowledgements}

This study is supported by the research grant FRGS/1/2014/SG04/04/UKM/01/1, sanctioned to Professor Dr. Ishak Hashim.

\section{References}

[1] W. Aung, G. Worku, Theory of fully developed, combined convection including flow reversal, J. Heat Transf., 108(1986), 299-304, DOI: $10.1115 / 1.3246958$

[2] A. J. Chamkha, On laminar hydromagnetic mixed convection flow in a vertical channel with symmetric and asymmetric wall heating conditions, Int. J. Heat Mass Transf., 45(2002), 2509-2525, DOI: 10.1016/S00179310(01)00342-8

[3] A. J. Chamkha, Non-Darcy fully developed mixed convection in a porous medium channel with heat generation/ absorption and hydromagnetic effects, Numer. Heat Transf. Part A, 32(1997), 653-675, DOI: 10.1080/10407789708913911

[4] A. J. Chamkha, T. Grosan, I. Pop, Fully developed mixed convection of a micropolar fluid in a vertical channel, Int. J. of Fluid Mech. Res., 30 (2003), 251-263, DOI: 10.1615/InterJFluidMechRes.v30.i3.10

[5] C. H. Cheng, H. S. Huang, and W. H. Huang, Flow reversal and heat transfer of fully developed mixed convection in vertical channels, J. Thermophys. Heat Transf., 4 (1990), 375-383, DOI: 10.2514/3.190

[6] S. U. S. Choi, Enhancing thermal conductivity of fluids with nanoparticles, ASME Fluids Eng. Div., 231 (1995), 99-105,

[7] M. M. Salah El-Din, Fully developed forced convection in a vertical channel with combined buoyancy forces, Int. Comm. Heat Mass Transf., 19 (1992), 239-248, DOI: 10.1016/0735-1933(92)90035-G

[8] T. Grosan, R. Pop, and I. Pop, Thermophoretic deposition of particles in fully developed mixed convection flow in a parallel-plate vertical channel, Heat Mass Transf., 45 (2009), 503-509, DOI: 10.1007/s00231-008-0443-z 
[9] A. Hadim, and G. Chen, Non-Darcy mixed convection in a vertical porous channel with asymmetric wall heating, J. Thermophys. Heat Transf., 8 (1994), 805-808, DOI: 10.2514/3.619

[10] M. Hajipour, and A. M. Dehkordi, Analysis of nanofluid heat transfer in parallel-plate vertical channels partially filled with porous medium, Int. J. Thermal Sci., 55 (2012), 103-113, DOI: 10.1016/j.ijthermalsci.2011.12.018

[11] M.H. Matin, and B. Ghanbari, Effects of brownian motion and thermophoresis on the mixed convection of nanofluid in a porous channel including flow reversal, Transp. Porous Med., 101 (2014), 115-136, DOI: $10.1007 / \mathrm{s} 11242-013-0235-\mathrm{x}$

[12] M. Mohammad, G. Ayub, and M.D. Asghar, Mixed-convection flow of nanofluids and regular fluids in vertical porous media with viscous heating, Ind. Eng. Chem. Res. 50 (2011), 9403-9414, DOI: 10.1021/ie2003895

[13] J. C. Umavathi, S. Veershetty, Non-Darcy mixed convection in a vertical porous channel with boundary conditions of third kind, Transp. Porous Med. 95 (2012), 111-131, DOI: 10.1007/s11242-012-0035-8

[14] J. C. Umavathi, J. P. Kumar, A. J. Chamkha, and I. Pop, Mixed convection in a vertical porous channel, Transp. Porous Med. 61 (2005), 315-335, DOI: $10.1007 / \mathrm{s} 11242-005-0260-5$. 
